\title{
Crystallization of a classical two-dimensional electron system: Positional and orientational orders
}

\author{
Satoru Muto and Hideo Aoki \\ Department of Physics, University of Tokyo, Hongo, Tokyo 113-0033, Japan
}

(Physical Review B59, 14911 (1999))

\begin{abstract}
Crystallization of a classical two-dimensional one-component plasma (electrons interacting with the Coulomb repulsion in a uniform neutralizing positive background) is investigated with a moleculardynamics simulation. The positional and the orientational correlation functions are calculated, to the best of our knowledge, for the first time. We have found an indication that the solid phase has a quasi-long-range (power-law) positional order along with a long-range orientational order. This indicates that, although the long-range Coulomb interaction is outside the scope of Mermin's theorem, the absence of ordinary crystalline order at finite temperatures applies to the electron system as well. The 'hexatic' phase, which is predicted between the liquid and the solid phases by the Kosterlitz-Thouless-Halperin-Nelson-Young theory, is also discussed.
\end{abstract}

Wigner pointed out in the 1930's that an electron system should crystallize due to the Coulomb repulsion for low enough densities. Although quantum effects are essential, the concept of electron crystallization can be generalized to classical cases. In fact, Grimes and Adams succeeded in observing a liquid-to-solid transition in a classical two-dimensional $2 \mathrm{D}$ ) electron system on a liquid-helium surface in 1979. In this system electrons obey classical statistics because the Fermi energy is much smaller than $k_{B} T$. The thermodynamic properties of the classical electron system are wholly determined by the dimensionless coupling constant $\Gamma$, the ratio of the Coulomb energy and the kinetic energy. Here $\Gamma \equiv\left(e^{2} / 4 \pi \epsilon a\right) / k_{B} T$, where $a=(\pi n)^{-1 / 2}$ is the meandistance between the electrons, $n$ the density of electrons, $e$ the charge of an electron, and $\epsilon$ is the dielectric constant of the substrate. For $\Gamma \ll 1$ the system will behave as a gas while for $\Gamma \gg 1$ as a solid. Grimes and Adams 2 found that the phase transition occurs at $\Gamma_{c}=137 \pm 15$.

On the other hand, Mermin proved rigorously, more than thirty years ago, that no true long-range crystalline orders are possible in the thermodynamic limit at finite temperatures in $2 \mathrm{D} .3 \mathrm{In}$ the proof, short-range interactions are assumed, while the $1 / r$ Coulomb interaction is too long-ranged to apply Mermin's arguments (see Ref. 3 for more precise mathematical conditions Although there have been some theoretical attempts 4 . the theorem to the long-range Coulomb case, no rigorous proof is attained up to now. Numerically, Gann et al.E investigated the problem with a Monte Carlo method by calculating the root-mean-square displacement. However they found it difficult to rule out the possibility that the root-mean-square displacement approaches a constant value in the thermodynamic limit, which is a conventional definition of a solid.

Another intriguing problem concerning 2D systems is the melting mechanism, where the Kosterlitz-ThoulessHalperin-Nelson-Young (KTHNY) theoryl 8 has predicted the existence of the 'hexatic' phase between the liquid and the solid phases (see Refs. 9, 10, 11 for re- views). The hexatic phase is characterized by a shortrange positional order and a quasi-long-range orientational order. Numerical calculations have been carried out by severalauthors with a molecular-dynamics (MD) method.12. 23.14 Most of the authors have obtained the melting point in good agreement with the Grimes-Adams experiment. However the results are rather controversial on the verification of the hexatic phase. Mor 12 concluded, from a MD result for the shear modulus, that the result agrees well mith the KTHNY theory. On the other hand, Kalia et al. 13 observed hysteresis in the temperature dependence of the total energy in their MD simulation to conclude that the melting is a first-order phase transition, which is incompatible with the KTHNY theory. The Monte Carlo study by Gann et al. 1 is also indicative of a first-order phase transition.

In addressing both of the above problems, i.e., the range of the ordering in the solid phase and the nature of the melting, the most direct way is to investigate the positional and the orientational correlation functions, since the phases should be characterized in terms of them. This is exactly the purpose of the present paper, which is done, to the best of our knowledge, for this system for the first time.

In order to accurately incorporate the temperature have employed Nosé-Hoover's canonical MD method 15 , 16 for the classical 2D electron system, while the previous calculations were done for micro-canonical ensemble.12,13.14 Electrons are confined to a rectangle with a rigid uniform neutralizing positive background. We impose periodic boundary conditions. An electron then interacts with infinite arrays of the periodic images with the long-range $1 / r$ potential. We employ the Ewald summation method to take care of this. The rectangle is chosen to be close to a square to minimize surface effects. The aspect ratio of the rectangle is taken to be $L_{y} / L_{x}=2 / \sqrt{3}$, which can accommodate a perfect triangular lattice 17 with $N=4 M^{2}$ ( $M$ : an integer) particles. The equations of motion are integrated numerically with Gear's predictor-corrector algorithm. We adopt a time 
step of $1.0 \times 10^{-12} \mathrm{sec}$, which guarantees six-digit accuracy in the energy conservation after several tens of thousands of steps.

We have performed the simulation both from a typical liquid $(\Gamma=60)$ and from a typical solid $(\Gamma=200)$. The initial conditions are set as follows: The electrons are placed randomly in the liquid phase or placed at the perfect triangular lattice points in the solid phase. The velocities of the electrons are assigned according to the Maxwell-Boltzmann distribution in either case. The lowest energy configurations are sought with a simulated annealing method. Namely, the positions and the velocities of the electrons are updated for a certain time interval. Once a thermal equilibrium sets in, the temperature is raised or lowered by a small amount. The latest positions and velocities are used as the initial conditions for the simulation at the new temperature. This procedure temporarily puts the system out of equilibrium, but updating the positions and velocities for a certain time interval equilibrates the system. This annealing process is repeated from a liquid phase to a solid phase (or vice versa) across the transition. We take care that the system is well equilibrated, especially near and after the transition, by allowing large numbers of time steps. The results presented in this paper are for $N=900$ electrons with MD runs from 30,000 to 110,000 time steps for each value of $\Gamma$. The correlation functions are calculated for the last 20,000 time steps.

Following Cha and Fertig 18 we define the positional and the orientational correlation functions from which we identify the order in each phase. First, the positional correlation function is defined by

$$
\begin{aligned}
C(r) & \equiv\left\langle\rho_{\mathbf{G}}^{*}(\mathbf{r}) \rho_{\mathbf{G}}(\mathbf{0})\right\rangle \\
& =\left\langle\frac{\sum_{i, j} \delta\left(r-\left|\mathbf{r}_{i}-\mathbf{r}_{j}\right|\right) \frac{1}{6} \sum_{\mathbf{G}} e^{\mathrm{i} \mathbf{G} \cdot\left(\mathbf{r}_{i}-\mathbf{r}_{j}\right)}}{\sum_{i, j} \delta\left(r-\left|\mathbf{r}_{i}-\mathbf{r}_{j}\right|\right)}\right\rangle,
\end{aligned}
$$

where $\mathbf{G}$ is the reciprocal vector of the triangular lattice, and $\rho_{\mathbf{G}}(\mathbf{r})=\exp (\mathrm{iG} \cdot \mathbf{r})$. The angular brackets in Eq. (1) stand for both the summation over particles and the thermal average. In Eq. (2) a summation is taken over six reciprocal vector G's that give the first peaks of the structure factor. In practice, the $\delta$-function must be broadened so that it can be handled numerically.

The orientational correlation function is defined by

$$
\begin{aligned}
C_{6}(r) & \equiv\left\langle\psi_{6}^{*}(\mathbf{r}) \psi_{6}(\mathbf{0})\right\rangle \\
& =\left\langle\frac{\sum_{i, j} \delta\left(r-\left|\mathbf{r}_{i}-\mathbf{r}_{j}\right|\right) \psi_{6}^{*}\left(\mathbf{r}_{i}\right) \psi_{6}\left(\mathbf{r}_{j}\right)}{\sum_{i, j} \delta\left(r-\left|\mathbf{r}_{i}-\mathbf{r}_{j}\right|\right)}\right\rangle,
\end{aligned}
$$

where $\psi_{6}(\mathbf{r})=\frac{1}{n_{c}} \sum_{\alpha}^{\text {n.n. }} e^{6 \mathrm{i} \theta_{\alpha}(\mathbf{r})}$, and $\theta_{\alpha}(\mathbf{r})$ is the angle of the vector connecting an electron at $\mathbf{r}$ and the $\alpha$-th nearest neighbor with respect to a fixed axis, say, the $x$-axis.
The summation is taken over $n_{c}$ nearestneighbors which are determined by the Voronoi diagram 19 , or equivalently its dual mapping, the Delaunay triangulation.

We first look at the positional and the orientational correlation functions for $\Gamma=200$ and $\Gamma=160$, typical solid phases, in Fig. 1. Although $\Gamma(T)$ is high (low) enough, the positional correlation is seen to decay slowly in both cases, indicating an algebraic decay at large distances. The round-off in the correlation function around half of the linear dimension of the system size is considered to be an effect of the periodic boundary conditions. The algebraic decay of the positional correlation function implies that the 2D electron solid has only a quasi-longrange positional order at finite temperatures. 20 Thus we have obtained a numerical indication that Mermin's theorem 3 applies to the electron system as well, which is consistent with the analytical (but not rigorous) results obtained in Refs. 4 , 5 .

By contrast, the orientational order is seen to be longranged. Therefore while the $2 \mathrm{D}$ electron solid has no true long-range crystalline order, it is topologically ordered. The triangular structure is seen as the peaks in both the positional and the orientational correlation functions (see the inset of Fig. 1).

We have plotted the Delaunay triangulation of a snapshot of the electron configuration for a solid $(\Gamma=160)$ or for a liquid $(\Gamma=90)$ in Fig. 2. We can in particular look at the topological defects, i.e., five-fold and seven-fold coordinated electrons. From the result the defects are seen to appear in isolated pairs, or more precisely in quartets, in the solid phase, which explains how a quasi-long-range positional order is compatible with a long-range orientational one. On the other hand, defects appear with a high density in the liquid phase.

We now focus on the orientational correlation function near the crystallization in Fig. 3. The result is obtained by cooling the system from a liquid to a solid. The orientational order is short-ranged for $\Gamma \leq 120$ and longranged for $\Gamma \geq 140$. Around the liquid-solid boundary $(\Gamma=130)$, the orientational correlation function, plotted on a double logarithmic scale in Fig. 3, indicates an algebraic decay (while the positional order is shortranged). The power evaluated from the data is approximately equal to unity, which is greater than the upper bound of $1 / 4$ predicted by the KTHNY theory. Large statistical errors near the transition, however, prevent us from drawing any definite conclusion on the existence of the hexatic phase. In fact, the correlation functions behave like a solid at $\Gamma=130$ when the system is heated from a solid to a liquid with no indication of the hexatic phase. This may be due to a finite-size effect, where a solid can be pinned in a melting process. For a small system, $N=100$, a solid phase in fact persists down to $\Gamma=120$ when heated.

Another finite-size effect is that, when the system crystallizes, the crystal axes can tilt from the unit cell axes of the finite system. The crystallization does occur in a tilted way in the present simulation. The misalignment 
causes a long relaxation time for the system to reach the lowest energy state. However the fact that the system crystallizes with tilted axes shows in itself that the $N=900$ system is sufficiently large in that boundary effects are not too strong. By contrast, we found that the crystal axes are always aligned to the unit cell for $N=100$. For the $N=100$ system, which is the size employed by Kalia et al.13, we found no indication of the hexatic phase, either. A numerical difficulty in MD simulations also arises from finite time steps. Finite time effects might result in insufficient equilibration, especially near a continuous transition. Even if the system is wellequilibrated, it would be difficult to tell a slow exponential decay from an algebraic decay in the correlation function for a finite system.

In summary, we have performed a molecular-dynamics simulation to investigate the ordering of a classical $2 \mathrm{D}$ electron system. From the positional and the orientational correlation functions we have found an indication that there is a quasi-long-range positional order and a long-range orientational order in the solid phase, which implies that Mermin's theorem is not spoiled even for the long-range $1 / r$ interaction. On the other hand, we have obtained only a sign, not a conclusive result, for the existence of the hexatic phase predicted by KTHNY theory, which thus remains an open question.

Although we have basically electrons on a liquidhelium surface in mind, a planar classical one-component plasma is recently realized as laser-cooled ions trapped in a disk region. 21 The disk has a finite thickness, for which stable crystalline phases are observed. If the ions could be trapped completely in $2 \mathrm{D}$, the present picture would be applicable. Conversely, it is an interesting theoretical problem to extend the present line of approach to planar systems with finite thicknesses.

We wish to thank Kazuhiko Kuroki, Hiroshi Imamura, Katsunori Tagami, and Naruo Sasaki for valuable discussions. The numerical calculations were mainly done with Fujitsu VPP500 at the Supercomputer Center, Institute for Solid State Physics, University of Tokyo.

${ }^{1}$ E. Wigner, Phys. Rev. 46, 1002 (1934).

${ }^{2}$ C. C. Grimes and G. Adams, Phys. Rev. Lett. 42, 795 (1979); D. S. Fisher, B. I. Halperin, and P. M. Platzman, ibid. B42, 798 (1979).

${ }^{3}$ N. D. Mermin, Phys. Rev. 176, 250 (1968).

${ }^{4}$ S. Chakravarty and C. Dasgupta, Phys. Rev. B22, 369 (1980).

${ }^{5}$ A. Alastuey and B. Jancovici, J. Stat. Phys. 24, 443 (1981).

${ }^{6}$ R. C. Gann, S. Chakravarty, and G. V. Chester, Phys. Rev. B20, 326 (1979).

${ }^{7}$ B. I. Halperin and D. R. Nelson, Phys. Rev. Lett. 41, 121 (1978); D. R. Nelson and B. I. Halperin, Phys. Rev. B19,
2457 (1979).

${ }^{8}$ A. P. Young, Phys. Rev. B19, 1855 (1979).

${ }^{9}$ B. I. Halperin, in Proceedings of Kyoto Summer Institute 1979 - Physics of Low-Dimensional Systems, edited by Y. Nagaoka and S. Hikami (Publication Office, Progress of Theoretical Physics, 1979), p. 53.

${ }^{10}$ D. R. Nelson, in Phase Transitions and Critical Phenomena, edited by C. Domb and M. S. Green (Academic Press London, 1983), Vol. 7, p. 1.

${ }^{11}$ K. J. Strandburg, Rev. Mod. Phys. 60, 161 (1988).

${ }^{12}$ R. H. Morf, Phys. Rev. Lett. 43, 931 (1979).

${ }^{13}$ R. K. Kalia, P. Vashishta, and S. W. de Leeuw, Phys. Rev. B23, 4794 (1981).

${ }^{14}$ R. W. Hockney and T. R. Brown, J. Phys. C8, 1813 (1975).

${ }^{15}$ S. Nosé, Mol. Phys. 52, 255 (1984); J. Chem. Phys. 81, 511 (1984).

${ }^{16}$ W. G. Hoover, Phys. Rev. A31, 1695 (1985).

${ }^{17}$ L. Bonsall and A. A. Maradudin, Phys. Rev. B15, 1959 (1977).

18 M.-C. Cha and H. A. Fertig, Phys. Rev. B50, 14368 (1994).

${ }^{19}$ M. P. Allen, D. Frenkel, W. Gignac, and J. P. McTague, J. Chem. Phys. 78, 4206 (1983).

${ }^{20}$ A power-law decay of the positional correlation function also implies that the Bragg spots in the structure factor should have a hexagonal symmetry with a power-law divergence of each spot in the thermodynamic limit.

${ }^{21}$ T. B. Mitchell, J. J. Bollinger, D. H. E. Dubin, X. P. Huang, W. M. Itano, R. H. Baughman, Science 282, 1290 (1998).

FIG. 1. The positional (the upper panel) and the orientational (the lower panel) correlation functions for $\Gamma=200$ and $\Gamma=160$. The horizontal scale is in units of the lattice constant of the triangular lattice. The inset in the lower panel illustrates the first four distances which give the peaks in the correlation functions.

FIG. 2. The Delaunay triangulation of a snapshot of the electron configuration for a solid with $\Gamma=160$ (a) or for a liquid with $\Gamma=90$ (b). Five(seven)-fold coordinated electrons are marked with empty (solid) circles. In (b) four(eight)-fold coordinated electrons are marked with empty (solid) squares. A part of the system is shown in either panel.

FIG. 3. The orientational correlation function near the crystallization. The result was obtained by cooling the system from a liquid to a solid. 


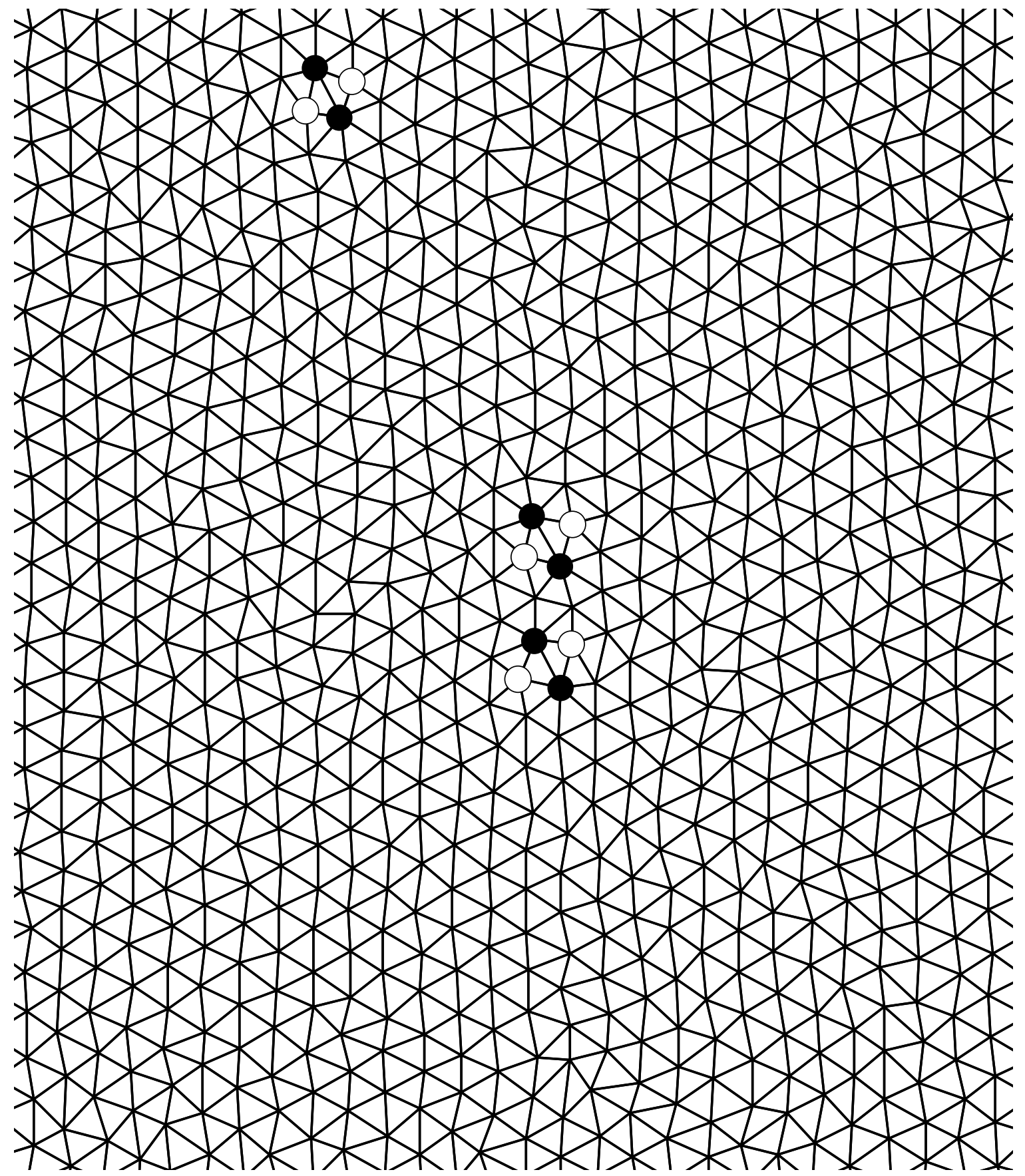

(a) 


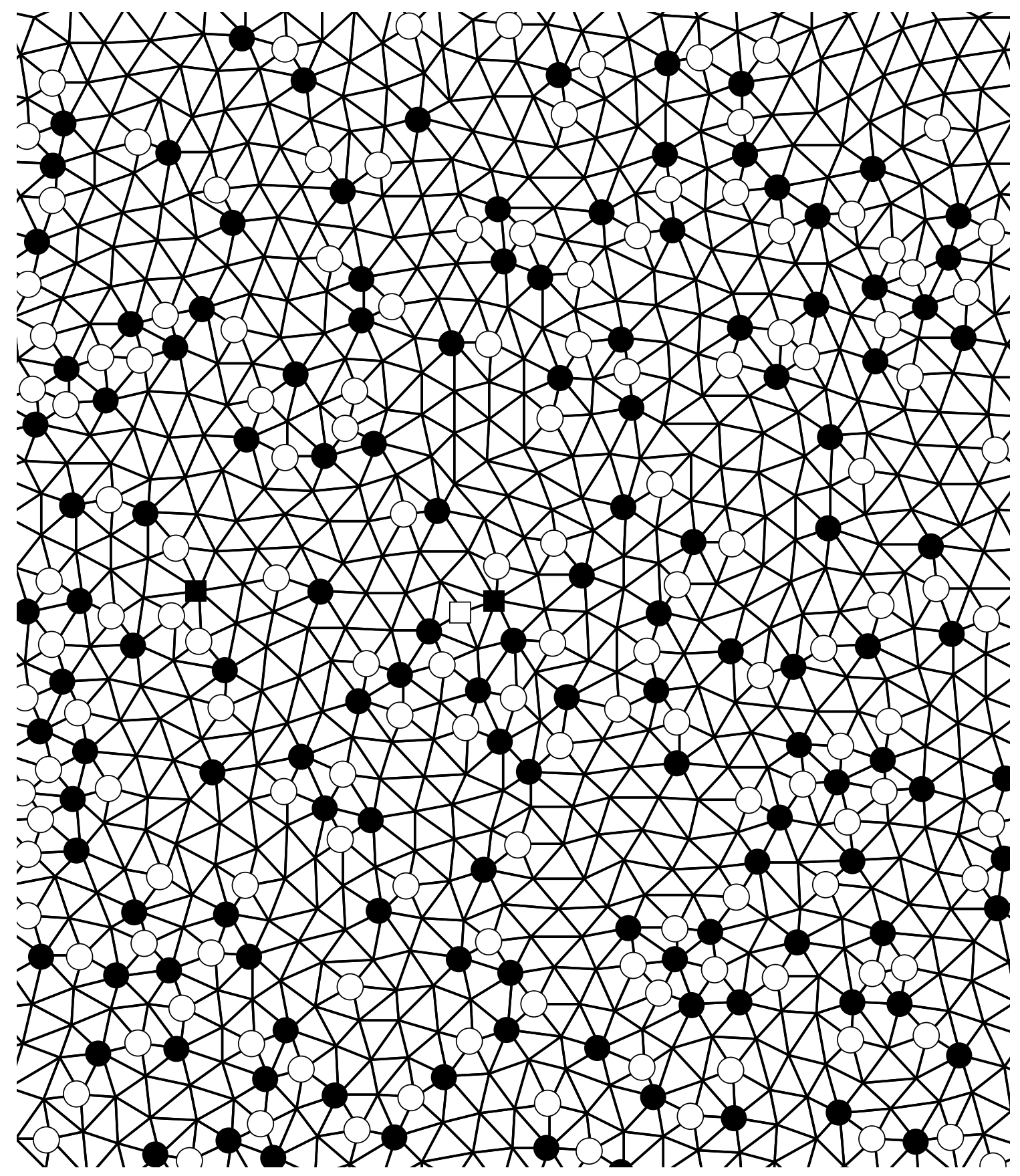

(b) 


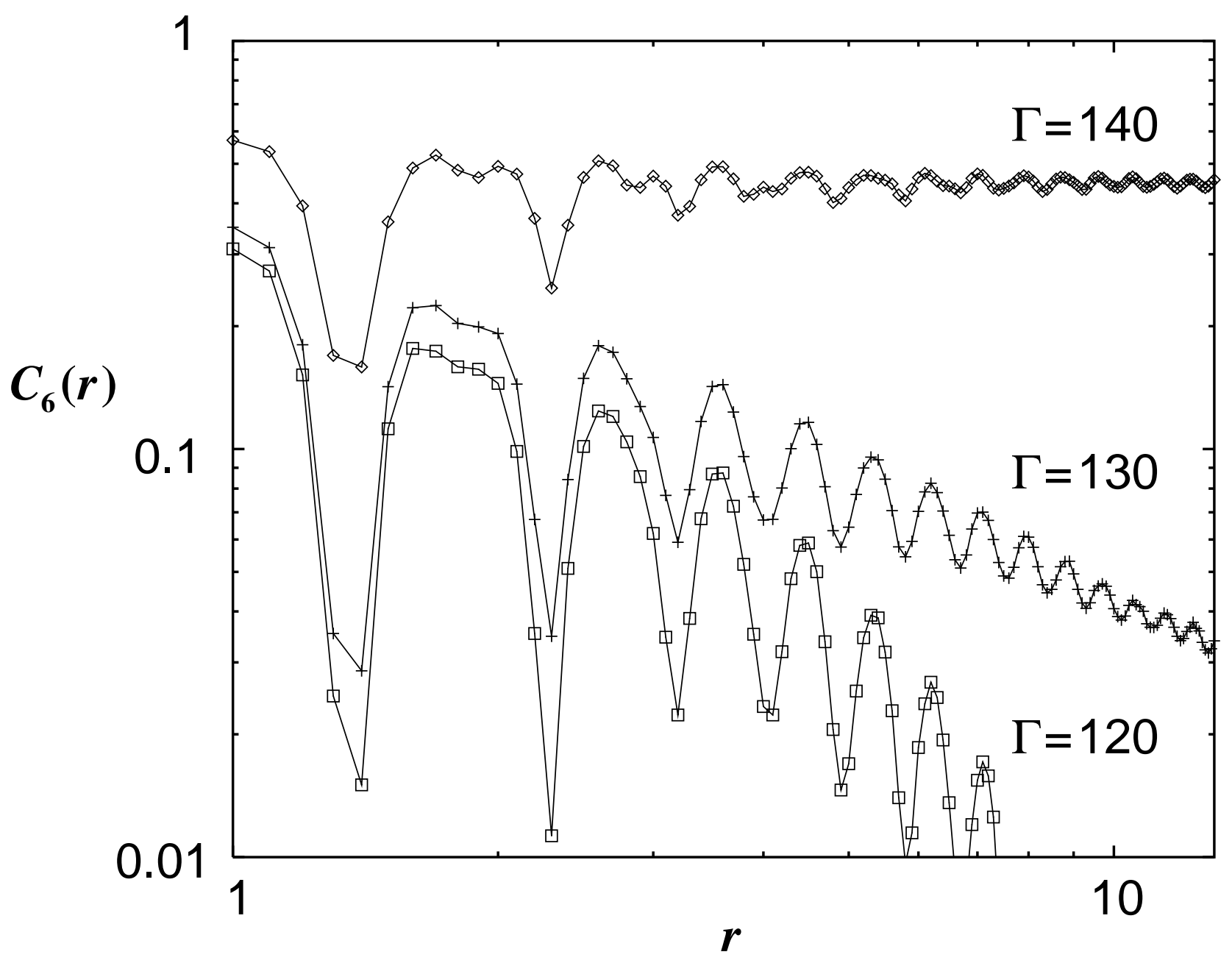

